\title{
Percutaneous Injection of Radiopaque Gelified Ethanol for the Treatment of Lumbar and Cervical Intervertebral Disk Herniations: Experience and Clinical Outcome in $\mathbf{8 0}$ Patients
}

\author{
(D) M. Bellini, D.G. Romano, S. Leonini, I. Grazzini, C. Tabano, M. Ferrara, P. Piu, L. Monti, and A. Cerase
}

\begin{abstract}
BACKGROUND AND PURPOSE: Chemonucleolysis represents a minimally invasive percutaneous technique characterized by an intradiskal injection of materials under fluoroscopic or CT guidance. Recently, a substance based on radiopaque gelified ethanol has been introduced. The purpose of this study was to describe the indications, procedure, safety, and efficacy of radiopaque gelified ethanol in the percutaneous treatment of cervical and lumbar disk herniations.
\end{abstract}

MATERIALS AND METHODS: Between September 2010 and August 2013, 80 patients (32 women and 48 men; age range, 18-75 years) were treated for 107 lumbar disk herniations ( $\mathrm{L} 2-\mathrm{L} 3, n=1 ; \mathrm{L} 3-\mathrm{L} 4, n=15 ; \mathrm{L} 4-\mathrm{L} 5, n=53$; and $\mathrm{L} 5-\mathrm{S} 1, n=38$ ) and 9 cervical disk herniations (C4-C5, $n=2 ; \mathrm{C} 5-\mathrm{C} 6, n=2 ; \mathrm{C} 6-\mathrm{C} 7, n=3$; and $\mathrm{C} 7-\mathrm{D} 1, n=2)$ by percutaneous intradiskal injection of radiopaque gelified ethanol under fluoroscopic guidance. Thirty-six patients underwent a simultaneous treatment of 2 disk herniations. Patient symptoms were resistant to conservative therapy, with little or no pain relief after 4-6 weeks of physical therapy and drugs. All patients were evaluated by the Visual Analog Scale and the Oswestry Disability Index.

RESULTS: Sixty-two of 73 (85\%) patients with lumbar disk herniations and 6/7 (83\%) patients with cervical disk herniations obtained significant symptom improvement, with a Visual Analog Scale reduction of at least 4 points and an Oswestry Disability Index reduction of at least $40 \%$. Leakage of radiopaque gelified ethanol in the surrounding tissues occurred in 19 patients, however without any clinical side effects.

CONCLUSIONS: In our experience, percutaneous intradiskal injection of radiopaque gelified ethanol is safe and effective in reducing the period of recovery from disabling symptoms.

ABBREVIATIONS: $\mathrm{CDH}=$ cervical disk herniation; $\mathrm{LDH}=$ lumbar disk herniation; $\mathrm{ODI}=$ Oswestry Disability Index; $\mathrm{RGE}=$ radiopaque gelified ethanol; $\mathrm{VAS}=$ Visual Analog Scale

C ervical disk herniation (CHD) and lumbar disk herniation (LDH) generally have a favorable natural history, often with long-term resolution of symptoms. ${ }^{1,2}$ However, pain can be debilitating, resulting in long absences from work or daily activities. Treatment is targeted at reducing both symptoms and the patient's recovery time. The first therapeutic approach is usually pharmacologic and physical; however, the clinical condition may not improve within a reasonable time. ${ }^{1}$

Received July 7, 2014; accepted after revision September 6.

From the Neuroimaging and Neurointerventional Unit - NINT (M.B., D.G.R., S.L., M.F., L.M., A.C.) and Unit of Neurology (P.P.), Department of Neurological and Neurosensorial Sciences, Hospital "Santa Maria alle Scotte," Siena, Italy; Department of Medical, Surgical and Neuro Sciences, Diagnostic Imaging (I.G.), University of Siena, Siena, Italy; and Unit of Radiology (C.T.), Hospital of Arzignano, Vicenza, Italy.

There has not been any financial support from commercial sources or pecuniary interest in such enterprises that could pose a conflict of interest, including consultations for any product or process mentioned in the submission.

Please address correspondence to Matteo Bellini, MD, Azienda Ospedaliera Universitaria Senese, Uoc NINT Neuroimmagini e Neurointerventistica, Viale Bracci n.16, 53100 Siena, Italy; e-mail: matteo.bellini@icloud.com

http://dx.doi.org/10.3174/ajnr.A4166
Chemonucleolysis is a minimally invasive percutaneous technique allowing intradiskal injection of various materials under fluoroscopic or CT guidance, to "digest" or dehydrate diskal portions. Materials include chymopapain, ${ }^{3}$ now abandoned because of frequent allergic reactions, ${ }^{4}$ and oxygen-ozone, ${ }^{5}$ whose results have still not been completely demonstrated. Recently, a newly developed substance, radiopaque gelified ethanol (RGE), has been introduced into clinical practice. ${ }^{6-10}$ RGE is a viscous solution containing ethyl alcohol and cellulose derivative products associated with a contrast agent (tungsten). Clinical outcomes seem to be encouraging, with a high reduction of possible collateral effects. ${ }^{4}$ The purpose of this article is to describe the results of our study in percutaneous intradiskal injection of RGE for $\mathrm{CDH}$ and $\mathrm{LDH}$.

\section{MATERIALS AND METHODS Population}

Between September 2010 and August 2013, 80 patients (32 women and 48 men; age range, $18-75$ years) were treated for 107 LDHs (L2-L3, $n=1$; L3-L4, $n=15$; L4-L5, $n=53$; and L5-S1, 
$n=38$ ), and 9, for CDH (C4-C5, $n=2$; C5-C6, $n=2$; C6-C7, $n=3$; and C7-D1, $n=2$ ) by percutaneous intradiskal injection of RGE. This retrospective evaluation of clinical and neuroradiologic data was in accordance with the rules of local institutional review boards.

\section{Indications}

Treatment was reserved for patients with recurrent drug-resistant neck or low back pain with brachialgia or sciatica (also incomplete) and no or minimal pain reduction after 4-6 weeks of physical therapy including resistive capacitive energy transfer, clinical neurodynamics, manual therapy, and global postural reeducation. Clinical evaluation included Lasègue, Slump, and Wassermann tests.

Sixty-seven patients underwent MR imaging; 13 patients underwent CT due to contraindications or claustrophobia. All patients with $\mathrm{LDH}$ showed at least 1 disk herniation at a metameric level consistent with symptoms. All patients with $\mathrm{CDH}$ and 18 with LDH underwent electromyography because of multiple disk herniations and uncertainty of the disk to be treated.

Inclusion criteria were disk herniations contained, without complete annulus tear, and not contained, with posterior longitudinal ligament integrity and no free fragments assessed with MR imaging $^{11}$ or CT. Because of the risk of adherences or epidural fibrosis, treatment was not performed in patients who had already undergone surgery at a clinical symptom level, with the exception of 1 patient with a recurrent LDH in whom gadolinium-enhanced MR imaging ruled out fibrous scar tissue.

Exclusion criteria were high disk degeneration (ie, the socalled "black disk" or a disk height loss of more than two-thirds), free isolated disk herniation fragments, stenosis of the intervertebral foramina or spinal canal, asymptomatic disk bulging, primary tumor or metastatic disease involving the level to be treated, active local or systemic infections, pregnancy, hemorrhagic diathesis, and anticoagulant therapy if not stopped before surgery.

$\mathrm{CDH}$ s were treated after neck color Doppler sonography, to rule out any anatomic variations or abnormalities of vessels, trachea, or esophagus.

All patients provided informed consent on the day before the procedure.

\section{Procedure}

Treatment was performed with the patient under local anesthesia, in "day-surgery" (71 patients) or short hospitalization (9 patients) regimens. If the patient was under antiplatelet or anticoagulant therapy, this was stopped 6 days before the procedure and converted to subcutaneous low-molecular-weight heparin.

Intravenous injection of short-term broad-spectrum antibiotic therapy including $2 \mathrm{~g}$ of amoxicillin/clavulanic acid (Augmentin) or $1 \mathrm{~g}$ of ceftriaxone disodium (Sirtap) was performed immediately before the procedure. Local anesthesia was by subcutaneous lidocaine 2\% (SELF SpA). Fluoroscopic guidance was by a digital biplanar rotational angiography (Innova 3131IQ; GE Healthcare, Milwaukee, Wisconsin). Under a sterile surgical regimen, a percutaneous approach was performed by using a spinaltype 20-ga needle (Spinocan; B. Braun Melsungen, Melsungen, Germany) for $\mathrm{CDH}$ and a Chiba 18-ga needle included in the RGE kit (DiscoGel; Gelscom SAS, Caen, France) for LDH. Needle insertion was monitored by fluoroscopic anteroposterior and lateral projections.

Patients with $\mathrm{CDH}$ were placed in a supine position, with a hyperextended neck. A right paravertebral approach was preferred due to a greater risk of esophageal lesions by a left approach. Lidocaine $2 \%(1-2 \mathrm{~mL}$ ) was injected in the superficial subcutaneous tissue. Medial to the right sternocleidomastoid muscle, the operator exerted sufficient pressure with the index and middle fingers of the left hand on the neck for lateral displacement of the common carotid artery and internal jugular vein, pushing the skin as close as possible to the intervertebral space, thus minimizing the needle tract and making the needle entry site "safe." Once penetrating the annulus, the needle was advanced until it reached the central portion of the disk.

Patients with $\mathrm{LDH}$ were placed in a prone position, usually by positioning an air-inflated cushion under the abdomen, to reduce the lumbar lordosis. Lidocaine $2 \%(5-10 \mathrm{~mL})$ was injected subcutaneously. The approach to the disks between L1 and L5 was posterior paravertebral, ipsilateral to the symptomatic side. Anatomic landmarks were identified by a $45^{\circ}$ lateral oblique projection, following the "scotty dog" technique. A Chiba needle was introduced at a paramedian entry point, $8-10 \mathrm{~cm}$ from the midline; it was directed parallel to the intervertebral space and anterior to the superior articular process (ie, "scotty dog" ear), to avoid puncturing the nerve root. The approach to the L5-S1 disk was technically more complex and partially limited by the iliac wing. At $45^{\circ}$, the needle was positioned with a high cranial C-arm inclination, tilted obliquely toward a narrow triangular area realized by the radiologic overlap of some skeletal structures, including the iliac wing, sacral wing, and corresponding superior articular process.

Intradiskal advancement of the needle was monitored by anteroposterior and lateral projections, because the tip has to reach the central portion of the nucleus pulposus. Crossing the annulus may result in a mild, hard elastic resistance, with possible patient's pain sensation, because it represents the only innervated zone. In all the LDHs, a mixture of triamcinolone acetonide (Triamvirgi) and ropivacaine $\mathrm{HCl}$ (Naropin) was injected near the nerve root, as a pharmacologic blockade, just before needle entrance in the disk.

\section{Preparation and Injection of RGE}

Inside its container, RGE is an attenuated liquid, which must be accurately shaken before being injected. The RGE solution should be injected slowly under continuous fluoroscopic guidance to check for extradiskal leakage (Fig. 1). The recommended dose is $0.8 \mathrm{~mL}$ for lumbar disks and $0.2 \mathrm{~mL}$ for cervical disks.

CT targeted to the treatment site was performed after the procedure in all the patients, with the exception of a young woman for radioprotection; this procedure was to verify the RGE distribution and rule out extradiskal leakage.

\section{Clinical Monitoring}

All patients were given the Visual Analog Scale (VAS) preoperatively and immediately after the treatment and 3 months later and a questionnaire concerning the quality of life based on the Os- 


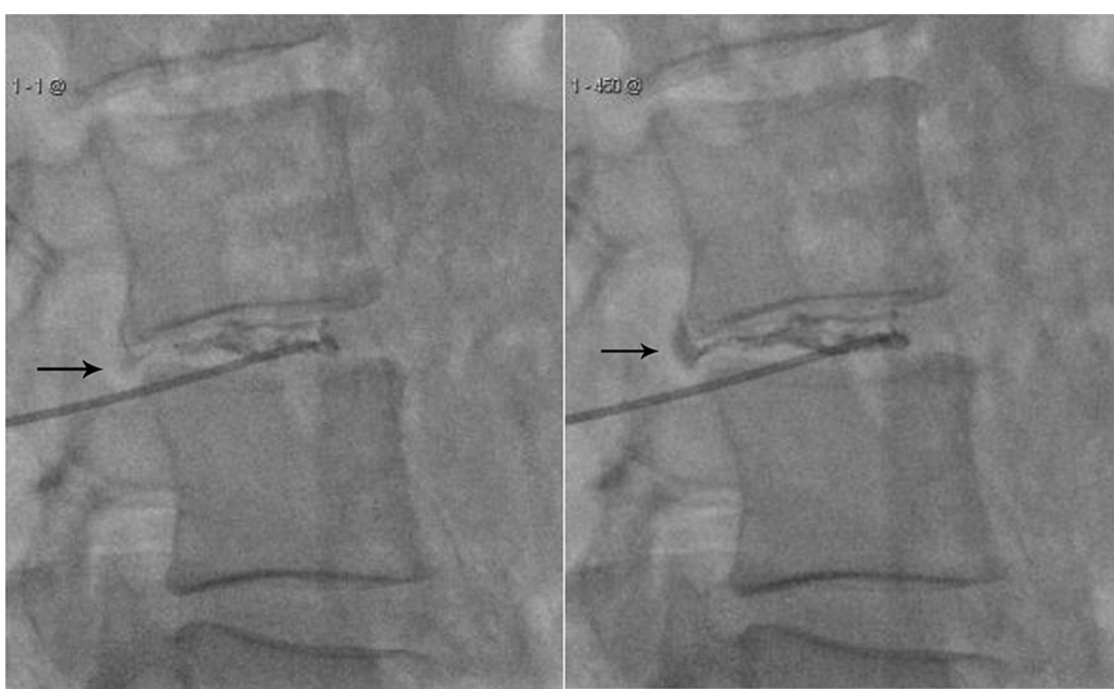

FIG 1. Progressive intervertebral disk filling of RGE. Real-time fluoroscopic images showing RGE inside the nucleus pulposus and in the herniated disk (arrows). Tungsten allows proper visualization of the material, including monitoring of possible leakage.

VAS scale

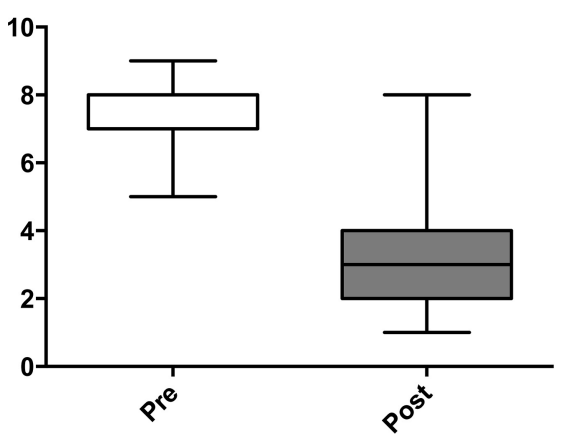

VAS scale

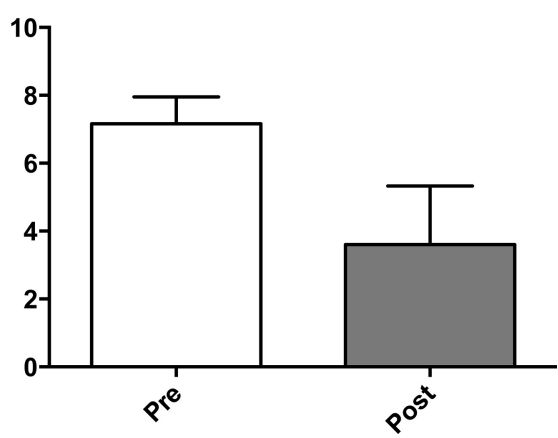

FIG 2. VAS scores recorded before and after percutaneous intradiskal injection of RGE. The left panel displays the boxplot of the data. Before treatment, the VAS scores were mostly concentrated on the upper values of the scale (median $=7 ; 25$ th percentile $=7 ; 75$ th percentile $=8$ ); therefore, this distribution was negatively skewed (skewness $=-0.29$ ). The distribution of the VAS scores in the posttreatment survey (median $=3 ; 25$ th percentile $=2 ; 75$ th percentile $=4$ ) became positively skewed (skewness $=0.91$ ). No outliers were identified. The reduction of 4 points in the median of the VAS scores between pre- and posttreatment was significant for the nonparametric Wilcoxon matched-pairs signed rank test $(W=-3076 ; P<.0001)$. The barplots in the right panel show the means and SDs of the data. The decrease of the means of the VAS scores from $7.16 \pm 0.79$ to $3.61 \pm 1.72$ was significant under the paired $t$ test with $78 d f(t=17.61 ; P<$ $.0001)$.

westry Disability Index $(\mathrm{ODI})^{12}$ before treatment and 3 months later.

\section{Statistical Analysis}

Analysis was performed on the VAS and ODI score values collected from all patients before treatment and at the 3-month follow-up. Their descriptive statistics such as mean, SD, median, and interquartile range are shown in Figs 2 and 3. To detect statistically significant change of the VAS and ODI in the posttreatment period compared with the pretreatment one, we used a paired $t$ test and Wilcoxon matched-pairs signed rank test. The null hypothesis of no difference between observed values before and after the treatment was then assumed for each series of scores. The series of ODI scores were labeled according to the canonical standing ${ }^{12}$ and then cross-tabulated to represent the pre- to postrank transi- tion. In correspondence to each rank detected before RGE injection and the percentage of the patients who have recovered after the treatment, the best rank (ie, minimal disability with the ODI score not $>20 \%$ ) was calculated.

Statistical analysis was assessed by commercial software (GraphPad Prism; GraphPad Software, San Diego, California; and Matlab; MathWorks, Natick, Massachusetts).

\section{RESULTS}

Thirty-four patients with LDH and 2 with $\mathrm{CDH}$ underwent simultaneous treatment of 2 disks. Seven patients with large LDHs either not contained or migrated were selected for RGE (Fig. 4).

Treatment was technically successful in all patients for all the 116 disk herniations (Figs 4 and 5). The procedure for a single disk required no more than 10-12 minutes from anesthesia to the end of the RGE injection. No relevant complications related to treatment occurred in any patient. There were no allergic reactions to RGE or other drugs. There were no cases of local or systemic iatrogenic infection.

Eight patients with LDH presented with temporary pain during treatment, locally or at the lower limb ipsilateral to the treated level, caused by passage of the needle close to a nerve root or during penetration of the annulus. In all these cases, symptoms resolved spontaneously a few minutes after treatment.

Postoperative CT showed a subtle extradiskal leakage of RGE in 14 patients with LDH (epidural, $n=2$; periradicular space, $n=4$; and posterior muscle tissues, $n=10)$. None reported any symptoms. In $62(85 \%)$ of the $73 \mathrm{pa}-$ tients treated for $\mathrm{LDH}$ and in $6(83 \%)$ of the 7 patients treated for $\mathrm{CDH}$, there was relevant pain reduction, usually within the first month after the procedure.

Statistical analysis indicated a significant difference in the scores between pre- and posttreatment conditions (Figs 2 and 3).

The mean decrease of the VAS index was -3.557 with a $95 \%$ confidence interval, 3.959 to -3.155 . The mean decrease of the ODI score was 30.38 with a $95 \%$ confidence interval, 33.79 to -26.96 .

The percentage of success was $>75 \%$ in the class of severe disability. Even 2 subjects in the sample with crippling back pain regained the minimal disability class. A reduction of at least 4 points in the VAS scale $(P<.0001)$ and at least $40 \%$ in the ODI score $(P<.0001)$ (Fig 2$)$ occurred in the first 2 months after 
ODI scale (\%)

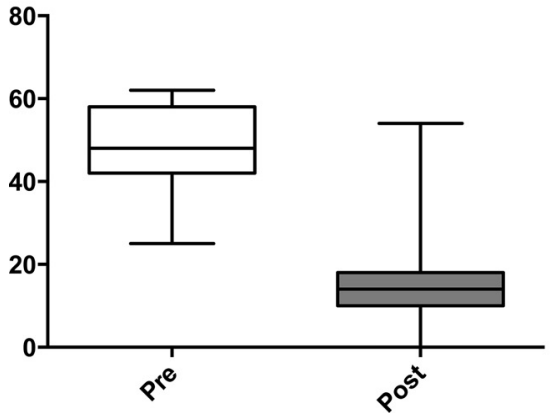

ODI scale (\%)

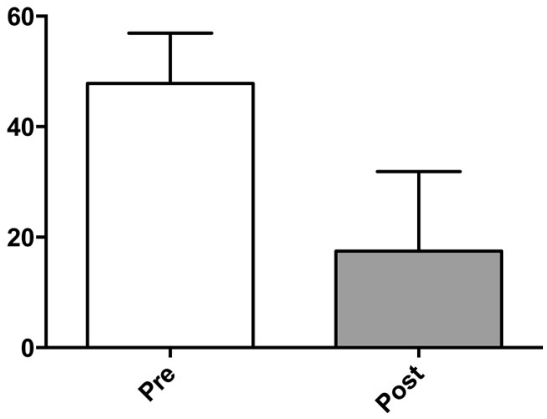

FIG 3. ODI scores recorded before and after percutaneous intradiskal injection of RGE. The index is expressed in percentage points and ranges from $0 \%$ to $100 \%$. The lower limit corresponds to the absence of disability; the upper limit, to the maximum degree of disability (patients are bed-bound). The left panel displays the boxplot of the data. Before treatment, the distribution of ODI scores (median $=48 \%$; 25 th percentile $=42 \%$; 75 th percentile $=58 \%$ ) was slightly negatively skewed (skewness $=-0.05)$, and it became positively skewed (skewness $=1.21$ ) in the posttreatment survey (median $=14 \%$; 25 th percentile $=10 \%$; 75 th percentile $=18 \%$ ). No outliers were identified. Between the pre- and posttreatment periods, the medians of the VAS scores dropped by 34 percentage points. This decline was significant for the nonparametric Wilcoxon matched-pairs signed rank test $(W=-3076 ; P<.0001)$. The barplots in the right panel show the means and SDs of the data. The decrease of the mean of the ODI scores from $47.85 \pm 9.05$ to $17.47 \pm 14.41$ was significant with the paired $t$ test with $78 d f(t=17.71, P<.0001)$.

treatment in $64(80 \%)$ patients and by the third month for the remaining 4 (5\%) patients who presented with large disk herniations, extruded or migrated (Fig 4). The patients reporting improvement resumed their normal activities within 1-3 months after treatment.

Twelve patients did not obtain relevant pain reduction within 6 months following treatment. Four underwent surgical diskectomy; the remaining 8 underwent other percutaneous treatments $(n=6)$ or tried other conservative therapy $(n=2)$, including anesthesiologist pain management.

\section{DISCUSSION}

DiscoGel (RGE) is a chemonucleolytic agent introduced for the first time in 2007 by Theron et al, ${ }^{6}$ containing ethyl alcohol, a cellulose derivative product, added to a radiopaque element (tungsten), causing dehydration of the nu-

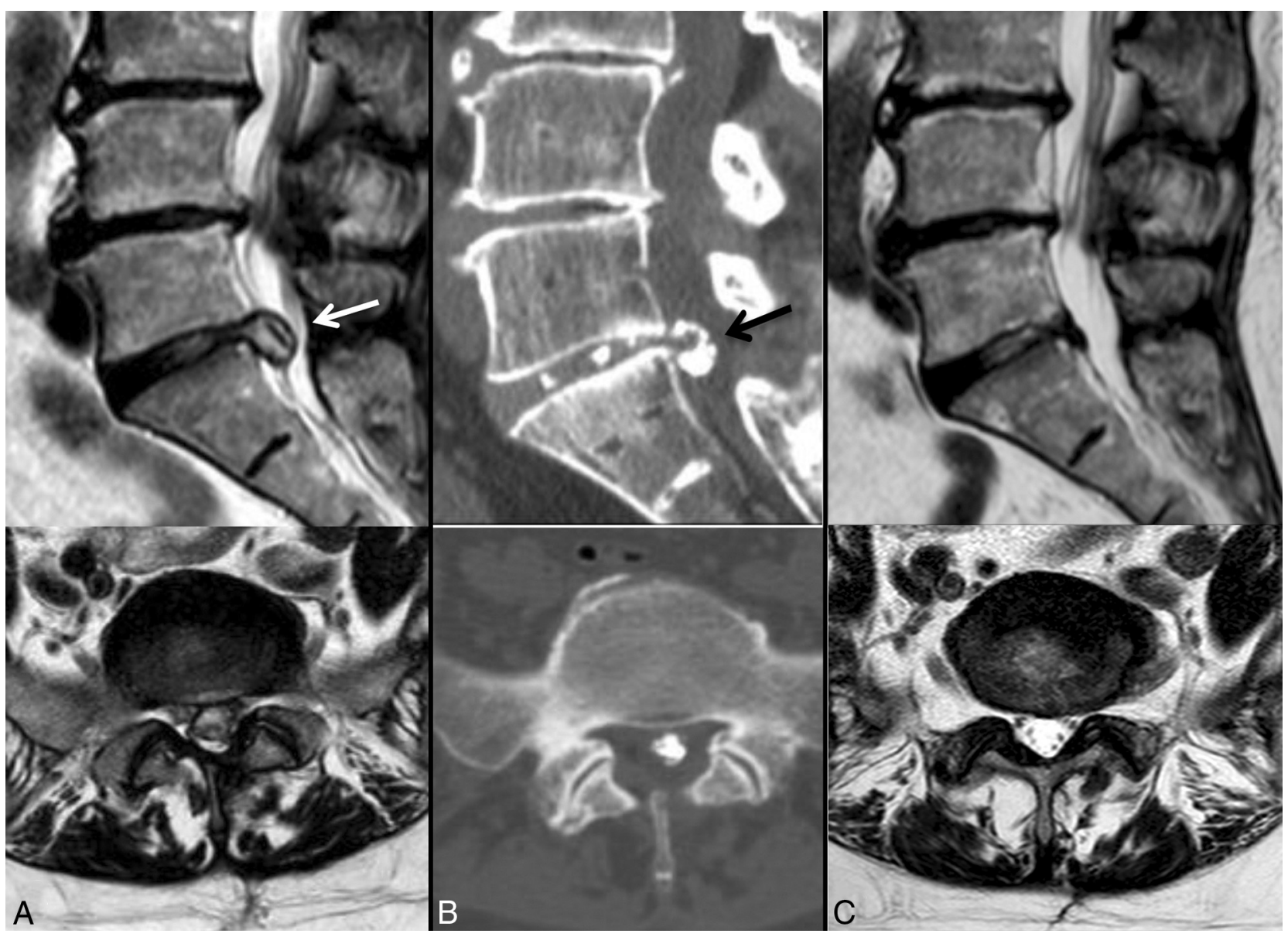

FIG 4. Treatment of a very large extruded LDH in a 58-year-old woman with left sciatica (VAS $=8$ ). $A$, In a preoperative MR imaging study, sagittal and axial T2 sequences show a large uncontained and partially migrated disk herniation at L5-S1 (median left posterolateral), with considerable root involvement (white arrow). B, CT control shows the optimal distribution of RGE in a disk herniation without leakage (black arrow). The patient did not have significant symptoms (VAS =3). C, MR imaging study obtained 6 months later shows complete dehydration and retraction of disk herniation. The patient was completely asymptomatic (VAS $=0)$. 

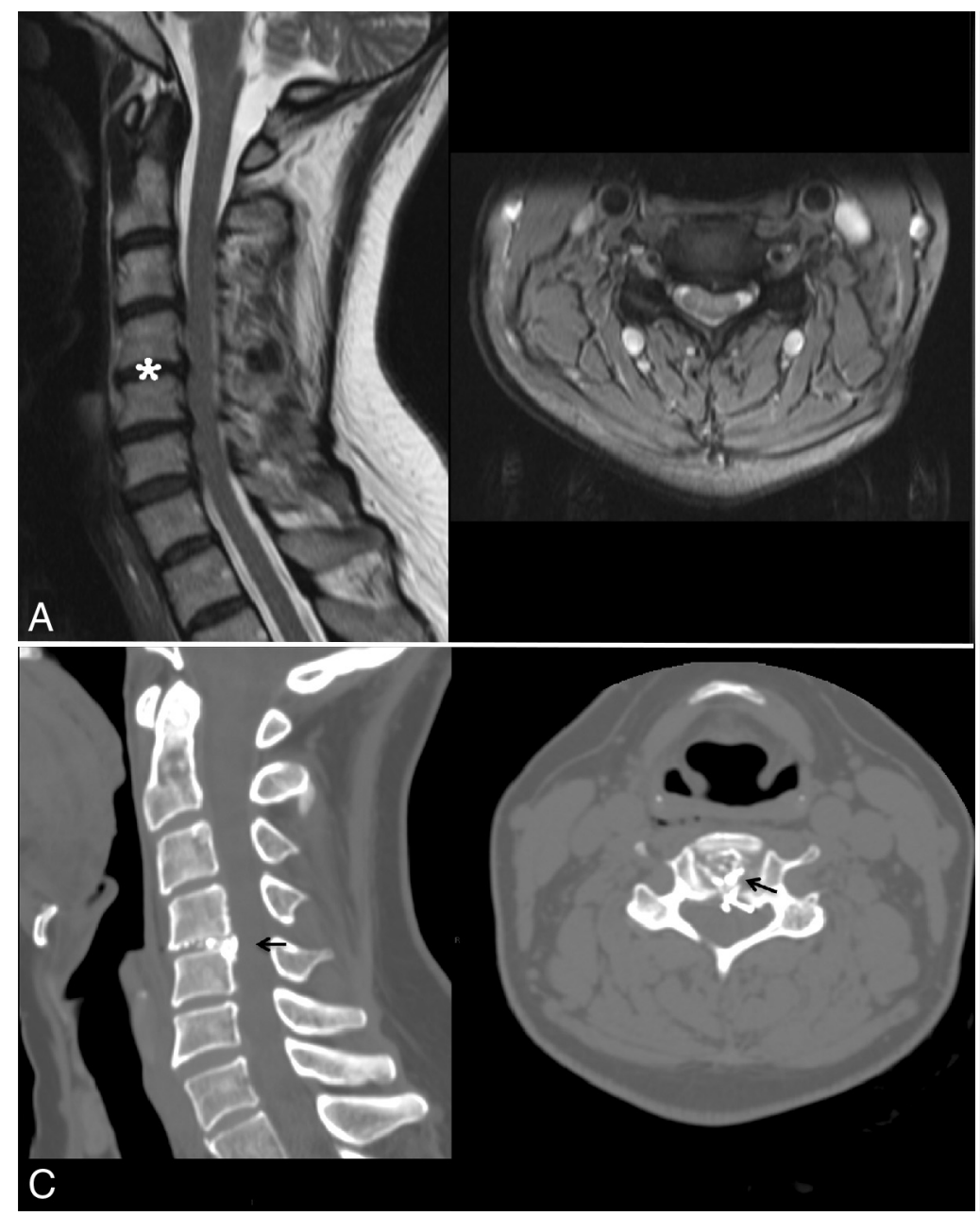

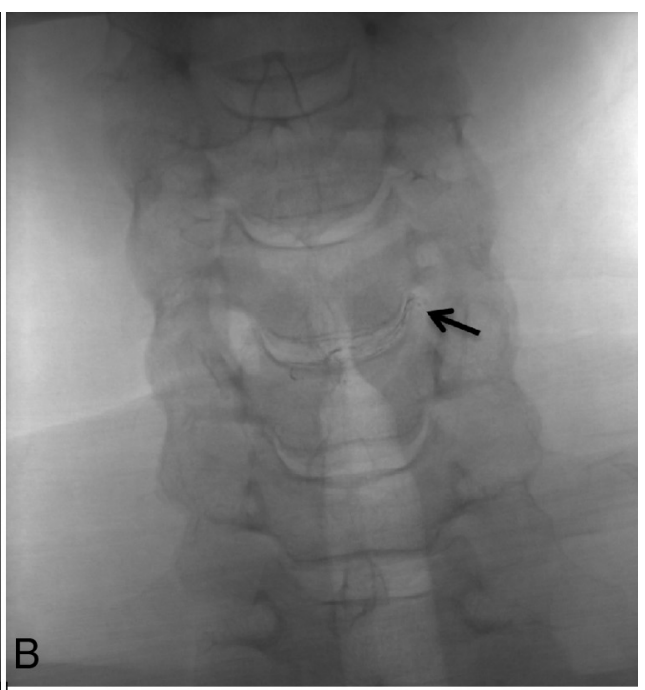

FIG 5. Treatment of a $\mathrm{CDH}$ in a 45-year-old woman with left brachialgia (VAS $=7$ ). $A$, T2-weighted sagittal and $\mathrm{T2}^{*}$-weighted axial MR images show a C4-C5 left posterolateral disk herniation. Note also other asymptomatic disk bulging at the $\mathrm{C} 3-\mathrm{C} 4$, C5-C6, and C6-C7 levels. Anteroposterior radiograph $(B)$ and $C T$ axial image $(C)$ obtained at the end of the procedure confirm the proper distribution of RGE in the treated disk, especially in the herniated portion (arrows). cleus pulposus and resulting in disk herniation retraction. In $\mathrm{LDH}$, Volpentesta et $\mathrm{al}^{10}$ reported excellent and good results in $80.4 \%$ of 72 patients, despite complications in $5.45 \%$. Theron et $\mathrm{al}^{6}$ reported a success rate of $91.4 \%$ in a group of 221 patients and a complication rate of $<0.5 \%$, while Stagni et $\mathrm{al}^{8}$ achieved a therapeutic success in 24 of 32 treated patients (75\%) without complications. In CDHs, Theron et $\mathrm{al}^{7}$ reported a success rate of $89.5 \%$ in 57 patients without complications.

Our experience has the following considerations.

Percutaneous intradiskal injection of RGE was simple and rapid, due to a biplanar digital rotational angiographic suite, which allowed an easier needle insertion, especially for $\mathrm{CDH}$. The procedure can also be executed by CT or CT fluoroscopy; however, the advantages of fluoroscopic guidance are known and include rapidity, real-time evaluation of RGE distribution and possible leakage, and dose reduction.

In both $\mathrm{LDH}$ and $\mathrm{CDH}$, we had no complications. Discomfort was minimal for most patients. They were questioned at the end of treatment and reported tolerating the procedure well. Even patients treated for $\mathrm{CDH}$ did not experience perceptible discomfort during the procedure, despite the lower amount of local anesthetic injected only at skin surface. Pain was greater in subjects with osteophytes or with a greatly reduced intervertebral space, making the needle insertion more complex, especially at the L5-S1 level.

We did not treat free fragments or highly degenerated disks.
Free fragments were separated from the disk and could not be reached by RGE injection. Highly degenerated disks, or "black disk" according to grade $\mathrm{V}$ of the Pfirrmann classification, are collapsed, without distinction between the nucleus and annulus, and are associated with reduced positive prognosis due to severe morphostructural changes. ${ }^{13}$ Moreover, RGE may result in further diskal thinning due to its chemonucleolytic effects. Additionally, we think that the gelling effect is reduced in highly degenerated disks because this process is directly correlated to the presence of water in the nucleus pulposus. In "black disks," radicular pain may also be related to marked reduction of the foraminal space in association with disk calcification and/or bulging or osteophytes. Thus, we usually prefer to perform a periradicular and intradiskal steroid and anesthetic injection and/or to propose an interspinous spacer implant.

We did not perform pretreatment diskography because an iodinated contrast agent may limit proper visualization of RGE, and RGE contacting fluids immediately gels, with the risk of occluding the needle or inducing a rapid premature gelling effect in the disk. Diskography has been performed before pure ethanol injection because of the relative difficulty in the control of its diffusion and leakage. RGE, instead, makes the alcohol solution much easier to handle and reduces the need for diskography. ${ }^{8}$ Notably, even in the largest herniations that are potentially "surgical," we did not consider the annulus integrity necessary, because RGE can spread 
to the more isolated disk compartments, leading to good results. In this subset of patients, the results have been encouraging, with convincing symptom improvement. Instead, verifying the annulus integrity is a critical factor in other percutaneous techniques, including mechanical or thermal decompression: These techniques are based on the principle of obtaining an annulus elastic return, especially at the herniated portion. This is possible only for contained disk herniations without an annulus tear.

The recommended dose of RGE is $0.8 \mathrm{~mL}$ for $\mathrm{LDH}$ and $0.2 \mathrm{~mL}$ for $\mathrm{CDH}$. In $\mathrm{CDH}$, the maximum recommended dose was always injected without leakage. In $\mathrm{LDH}$, the amount ranged from 0.5 $\mathrm{mL}$ to $0.8 \mathrm{~mL}$, depending on the disk space amplitude and relative disk capacity to accommodate the RGE and to avoid important leakages. We always stopped injection at the observation of initial epidural or foraminal space leakage. It is also important to avoid any possible irritative effect on soft paravertebral tissues, though it is well-demonstrated that no morphostructural changes were caused by intradiskal, intraforaminal, and epidural RGE. ${ }^{14}$

Technically, $>2$ levels could be treated in the same session, in the absence of specific recommendations by the manufacturer. Three treated levels in a patient but in 2 separate sessions have been recently reported. ${ }^{10}$ Currently, the literature does not report a case of 4 disks treated. The main reasons are that most patients would not tolerate a simultaneous multilevel treatment in the same procedure, and an increased number of treated levels would heighten the risk of complications and exposure to x-rays for both patient and operator. Additionally, preliminary clinical and neuroradiologic evaluation in most cases established the levels to be treated. Finally, we agree with those authors ${ }^{10}$ who propose treating $>2$ levels in different sessions, to verify whether the first treatment was the most appropriate.

RGE leakage occurred in extradiskal tissues, in the epidural space, or in the periradicular space, after LDH treatment; this was caused by reflux through the trajectory of the needle. It has been asymptomatic and reported by other authors. ${ }^{14}$ In daily clinical practice, we agree to propose follow-up CT only in patients with unchanged or worsening symptoms, to better understand the RGE distribution.

In most patients, clinical improvement occurred during the first month after treatment. Improvement occurred earlier in $\mathrm{CDH}$ than in LDH, despite the lower amount of drugs. In LDH, patients with a faster reduction in symptoms were those with a single herniation and treated at the L4-L5 or L5-S1 levels without other associated factors (ie, canal or foramina stenosis). Only in cases of larger and extruded herniated disks and in recurring herniation did improvement take longer (approximately 3 months).

Clinical benefits could be partially due to the gelling effect of RGE that, spontaneously engaging the herniation breach, should prevent a greater diskal excursion under axial loading. This hypothesis might be demonstrated by pre- and postoperative orthostatic spinal MR imaging. ${ }^{15}$ Possible, but not proved, is the potential effect of ethanol on the reduction of local inflammatory mechanisms generated by the herniation itself (ie, mediated by the release of cytokines and other endogenous substances). This may affect, in part, the favorable development of such treatment clinically. ${ }^{1}$

In Europe, the cost of RGE is higher than that of other chemonucleolytic agents, like oxygen-ozone; however, it is similar to that of nucleoplasty or decompressive treatment. In our hospital, the cost is less than a third of that of a single-level surgical diskectomy. Additionally, compared with surgery, percutaneous RGE may take only 1 day of hospitalization.

\section{CONCLUSIONS}

In our experience, percutaneous RGE for the treatment of $\mathrm{CDH}$ and $\mathrm{LDH}$ was safe and reduced morbidity, with good clinical results (in some cases even in the very early stages after the treatment). Due to its minimally invasive nature, this technique reduced the recovery period from the disabling symptoms typical of disk herniations.

\section{ACKNOWLEDGMENTS}

We thank Prof. Alessandro Rossi, chief of the Neurologic and Neurosensorial Sciences Department ("Santa Maria alle Scotte” University Hospital, Siena, Italy), for his invaluable help and comments.

Disclosures: Alfonso Cerase-UNRELATED: Board Membership:Journal of Pediatric Neuroradiology (editorial board).

\section{REFERENCES}

1. Benoist $M$. The natural history of lumbar disc herniation and radiculopathy. Joint Bone Spine 2002;69:155-60

2. Bozzao A, Gallucci M, Masciocchi C, et al. Lumbar disk herniation: MR imaging assessment of natural history in patients treated without surgery. Radiology 1992;185:135-41

3. Nordby EJ, Wright PH. Efficacy of chymopapain in chemonucleolysis: a review. Spine (Phila Pa 1976) 1994;19:2578-83

4. Sussman BJ. Inadequacies and hazards of chymopapain injections as treatment for intervertebral disc disease. J Neurosurg 1975;42:389-96

5. Muto M, Andreula C, Leonardi M. Treatment of herniated lumbar disc by intradiscal and intraforaminal oxygen-ozone (O2-O3) injection. J Neuroradiol 2004;31:183-89

6. Theron J, Guimaraens L, Casasco A, et al. Percutaneous treatment of lumbar intervertebral disk hernias with radiopaque gelified ethanol: a preliminary study. J Spinal Disord Tech 2007;20:526-32

7. Theron J, Cuellar H, Sola T, et al. Percutaneous treatment of cervical disk hernias using gelified ethanol. AJNR Am J Neuroradiol 2010;31:1454-56

8. Stagni S, de Santis F, Cirillo L, et al. A minimally invasive treatment for lumbar disc herniation: DiscoGel ${ }^{\circledR}$ chemonucleolysis in patients unresponsive to chemonucleolysis with oxygen-ozone. Interv Neuroradiol 2012;18:97-104

9. de Sèze M, Saliba L, Mazaux JM. Percutaneous treatment of sciatica caused by a herniated disc: an exploratory study on the use of gaseous discography and Discogel $\left({ }^{\circledR}\right)$ in 79 patients. Ann Phys Rehabil Med 2013;56:143-54

10. Volpentesta G, De Rose M, Bosco D, et al. Lumbar percutaneous intradiscal injection of radiopaque gelified ethanol ("Discogel") in patients with low back and radicular pain. J Pain Relief 2014;3:145

11. Oh KJ, Lee JW, Yun BL, et al. Comparison of MR imaging findings between extraligamentous and subligamentous disk herniations in the lumbar spine. AJNR Am J Neuroradiol 2013;34:683-87

12. Fairbank JC, Pynsent PB. The Oswestry Disability Index. Spine (Phila Pa 1976) 2000;25:2940-52; discussion 2952

13. Mwale F, Iatridis JC, Antoniou J. Quantitative MRI as a diagnostic tool of intervertebral disc matrix composition and integrity. Eur Spine J 2008;17(suppl 4):432-40

14. Guarnieri G, De Dominicis G, Muto M. Intradiscal and intramuscular injection of Discogel ${ }^{\circledR}$ : radiopaque gelified ethanol—pathological evaluation. Neuroradiology J 2010;23:249-52

15. Tarantino U, Fanucci E, Iundusi R, et al. Lumbar spine MRI in upright position for diagnosing acute and chronic low back pain: statistical analysis of morphological changes. J Orthop Traumatol 2013; $14: 15-22$ 